\title{
An incidental finding of absent gall bladder in laparoscopic exploration - a rare occurrence in extra hepatic biliary anatomy
}

I.H.D.S. Pradeep ${ }^{1}$, I.H.D.S. Prasad ${ }^{1}$, D. Wickramarathna ${ }^{1}$, C.A.H. Liyanage ${ }^{2}$

1 Professorial Surgical Unit, North Colombo Teaching Hospital, Ragama, Sri Lanka

2 Department of Surgery, University of Kelaniya, Sri Lanka

\section{Introduction}

Congenital agenesis of the cystic duct and gall bladder is one of the rare occurrences seen in extra hepatic biliary anatomy. It represents a variation in the process of embryogenesis of extra hepatic biliary tree with the incidence of $0.01 \%-0.06 \%$ [1]. This itself can present as an isolated occurrence or associated with other congenital malformations of the extra hepatic biliary tree and the liver [2]. Half of the patients present with symptoms similar to biliary colic or chronic cholecystitis despite an absent gall bladder [10].

\section{Case Report}

A 53 year old previously healthy female was investigated for right hypochondrial pain of 6 months duration. Pain was dull and aching in nature and there was no radiation. It was associated with dyspeptic symptoms and aggravated following ingestion of meals with high fat content. There were no symptoms of obstructive jaundice, biliary colic or chronic constipation.

Patient was anicteric with a soft, non-tender abdomen and there were no organomegaly or palpable masses detected on routine abdominal examination. Her liver function tests and Upper GI endoscopy were normal. Ultrasonography of the abdomen revealed a contracted gall bladder with wall thickening. Considering the above facts a clinical diagnosis of chronic cholecystitis was made and she was scheduled for laparoscopic cholecystectomy due to persistent symptoms.

She underwent laparoscopic exploration which revealed the absence of the gall bladder within gall bladder fossa. Laparoscopy was converted to laparotomy for further exploration. The common bile

Correspondence: I.H.D.S Pradeep

E-mail: samaniddagoda@gmail.com duct was explored in full length and the gall bladder and the cystic duct could not be identified. She had an uneventful post-operative period.

She was scheduled for post-operative Endoscopic Retrograde Cholangiopancreatography (ERCP) which confirmed the diagnosis of absent gall bladder and cystic duct with normal intra hepatic ducts and extra hepatic bile ducts. Extra hepatic location of the gallbladder could not be identified. (Figure 1a, 1b)

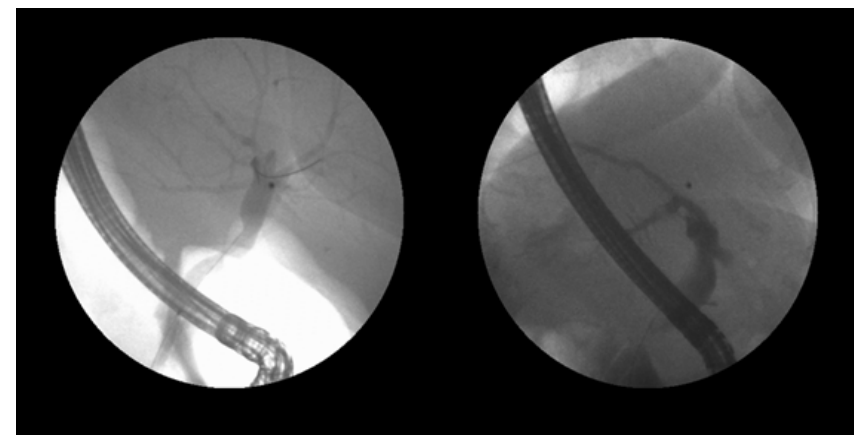

Figure 1a, 1b.

\section{Discussion}

In embryogenesis, the liver and extra hepatic biliary system begins to develop in early intrauterine life as a ventral out pouching from the caudal part of the foregut. Any aberrations of the intrauterine developmental process results in agenesis of the gall bladder and other associated abnormalities of the extra hepatic biliary tree [2].

Gall bladder agenesis has been described as 3 clinical entities [1]:

- Symptomatic $(50 \%)$,

- Asymptomatic (an incidental finding during laparoscopy or laparotomy) (35\%)

- Associated with multiple fetal anomalies (15\% to $16 \%$ ). 
Symptomatic patients usually presents with clinical features of chronic cholecystitis or biliary colic which is further confirmed ultrasonologically as contracted or shrunken gall bladder with or without biliary calculi. These patients undergo surgery due to recurrent complaints of gall bladder symptoms supported by the ultrasound scan report which mimics chronic cholecystitis [4].

Shrunken, contracted gall bladder of chronic cholecystitis mimics the radiological appearance of agenesis of the gall bladder and this is the most frequent ultrasonography seen in patients later found to have agenesis of the gall bladder [7]. Non-visualization of the gall bladder during pre-operative abdominal ultrasound scan in symptomatic patients is commonly attributed to an obstructed cystic duct, anatomic variations, inadequate fasting or technical error. Possibility of congenital absence of gall bladder is rarely considered.

Due to the presence of wide variations in extra hepatic biliary anatomy, an abnormal location of the gall bladder has to be excluded. Potential sites of ectopic gall bladder are intra-hepatic, left-sided, beneath the posterior inferior surface of liver, between the leaves of the lesser omentum, within the falciform ligament, retroperitoneal, retro hepatic, or in the retro pancreatic and retro duodenal areas [3].

In doubtful cases, further imaging should be obtained before operative interventions $[1,6]$. Computed tomography (CT) scanning, Magnetic resonance cholangiopancreatography (MRCP) and ERCP can be used to diagnose agenesis of the gall bladder pre operatively in doubtful cases. [11]

In patients who have been diagnosed during surgery, intra operative findings should be confirmed post operatively using ERCP or MRCP to visualize the extra hepatic and intrahepatic biliary anatomy. Extensive surgical exploration is not recommended [7, 10].

\section{Conclusion}

Agenesis of the gall bladder is a rare congenital anomaly.
Clinical and radiologic features mimic chronic cholecystitis, and patients frequently undergo unnecessary operative procedures. In cases which are diagnosed intra-operatively, extensive surgical exploration is not indicated [7]. Further radiological evaluation should follow during post-operative period to confirm the diagnosis. A high index of suspicion is needed by surgeons and radiologists to diagnose this entity preoperatively. There are no specific guidelines for management of symptoms in gall bladder agenesis; conservative management with smooth muscle relaxants is indicated [10].

\section{References}

1.Bennion RS, Thompson JE, Tompkin RK. Agenesis of the gallbladder without extrahepatic biliary atresia. Arch Surg 1988, 123:1257-1260.

2. Turkle SB, Swanson V, Chandrasoma P. Malformations associated with congenital absence of the gallbladder. J Med Genet 1983, 20:445-449

3. Sherson ND. The absent adult gallbladder. Aust N Z J Surg 1970, 39:225-261.

4. Kabiri H, Domingo OH, Tzarnas CD. Agenesis of the gallbladder.Curr Surg 2006, 63:104-106.

5. Serour F, Klin B, Strauss S, Vinograd L. Agenesis of gallbladder revisited laparoscopically. Surg Laparosc Endosc 1993, 2:144-146.

6.Grandhi TM, El-Rabaa SM. Agenesis of the gall bladder and cystic duct: laparoscopic diagnosis. Int J Gastroenterol 2005, 4(1)

7.Balakrishnan S, Singhal T, Grandy-Smith S, El-Hasani S. Agenesis of the gallbladder: lessons to learn.JSLS 2006, 10:517-519.

8. Joliat GR, Shubert CR, Farley DR .Isolated congenital agenesis of the gallbladder and cystic duct: report of a case. J Surg Educ. 2013 Jan-Feb; 70(1):117-20.

9. Aspevik RK1, Hjelseth B, Irtun. [Agenesis of the gallbladder]. Tidsskr nor laegeforen. 2002Nov 30; 122(29):2774-6.

10.Pashtoon Murtaza Kasi, Raymund Ramirez, Shari. Rogal, Kailey Littleton, and Kenneth. Fasanellac.Gallbladder Agenesis. Case Rep Gastroenterol. 2011 Sep-Dec; 5(3): 654-662.

11. Fiaschetti V, Calabrese G, Viarani S, Bazzocchi G, Simonetti G. Gallbladder agenesis and cystic duct absence in an adult patient diagnosed by magnetic resonance cholangiography: report of a case and review of the literature. Case Report Med. 2009; 2009:674-768. 


\section{Key Points:}

- Patients with features of chronic cholecystitis or biliary colic are incidentally found to have the rare congenital anomaly of Agenesis of the gallbladder.

- Computed tomography (CT) scanning, Magnetic resonance cholangiopancreatography (MRCP) and Endoscopic retrograde cholangiopancreatography (ERCP) can be used to diagnose agenesis of the gallbladder preoperatively. 